\title{
Acting Up or Opting Out: An Analytical Literature Review of Extant South African and International School Truancy Studies
}

\author{
Maynard van Breda \\ College of Education, University of South Africa, South Africa \\ Email:vbredmj@unisa.ac.za
}

\section{Doi:10.5901/mjss.2014.v5n16p322}

\section{Abstract}

\begin{abstract}
The common purpose of this article is to review recent international and South African research on school truancy and the implementation of intervention strategies. The specific aim is to examine the effects of interventions on school attendance in order to inform policy, practice and research. Consequently, this review is limited to a consideration of definitional issues, causes of truancy and non-attendance and on recent trends in truancy intervention research. Much of the research draws attention to the fact that there is a diversity of views as to causal factors of this behaviour. Some suggest that school truancy is the effect of dysfunctional family backgrounds and home life, education systems which fail learners, unjust social and economic systems as well as psychological traits among learners such as low level of self-esteem and poor self-concept. The article concludes with a discussion on intervention strategies aimed at reducing the prevalence of truant behaviour. It is also envisaged that the implications of this review will provide evidence, guidance as well as some caution for future researchers who wish to advance the study of truant behaviour among students and the implementation of intervention strategies.
\end{abstract}

Keywords: truancy; absenteeism; intervention programs;

\section{Introduction}

Playing truant used to be a one-time lark for many school going students. More often than not, these antics were shortlived as local shopkeepers, neighbours and family friends were quick to report truants to parents or school authorities. However, the past three to four decades, saw increasing evidence and an evolving recognition that school truancy is fast growing into a profound problem and major concern locally and internationally. Truant behaviour has become an issue that has incrementally been identified as one of the risk factors that can be linked to delinquent activity, substance abuse and educational failure (Ovink, 2011; Hendricks, Sale, Evans, Mckinley and Carter, 2010). In many instances it may signal the beginning of a lifetime of challenges for students who choose to skip classes deliberately and often do not realise the negative repercussions of their behaviour. These students are likely to fall extremely far behind in their school work and many of them eventually drop out, since in their judgement, dropping out of school is comparatively speaking much easier than catching up.

Despite numerous anti-truancy campaigns and social work intervention launched internationally to address the issue of school truancy, there seems to be a broadening body of evidence among researchers that poor school attendance and skipping classes intermittently not only has substantial cost implications for the individual alone, but also for the wider society (Maynard, McCrea, Pigott and Kelly, 2013; Valentine, Pigott and Rothstein, 2009). Not only does it cost students an education, but invariably results in limited job opportunities and earning power, thereby restricting chances for future education and training. The implications for schools with high rates of absenteeism and truancy related challenges include loss of funds, failure to meet performance requirements and targets set by local, provincial and national education authorities. Significant costs to communities associated with truancy include higher rates of criminal activity, citizens not productively contributing to the community as well as higher government spending for social services (Goldstein, Little and Akin-Little, 2003). Other potentially risky outcomes associated with truancy include delinquent activity, substance abuse, gang-related activity, involvement in criminal behaviour such as burglary, vehicle theft and school expulsion (Maynard, Salas-Wright, Vaughn \& Peters, 2012; Petrides, Chamorro-Premuzic, Frederickson \& Furnham, 2005; Reid 2002).

Reid (1994) contended that in the United States of America it is estimated that approximately $55 \%$ of students in secondary schools either do not attend school on a regular basis or skip classes regularly during the day. According to this author, many teachers of truanting students, being only human, are often not too concerned when they find only 20 out of the regular 30 students present in their classes. Evidently, such a situation simply translates into less work, less 
head ache and more manageable units to work with. Reid (1994) further emphasized that in the American context, the majority of persistent truants are students who are not performing well at school, lack parental encouragement and who face a variety of material or social challenges which are often unrelated to school. Unlike in America and in European countries where extensive truancy studies have been conducted, the exploration of this behavioural phenomenon has remained relatively limited in South African.

As is the case internationally, the tendency by students to skip certain classes particularly towards the end of the school day in South African schools, demonstrates that some form of "hidden truancy" is prevalent and that students can be marked present in the school's attendance register, but fail to attend all their lesson. On the other hand, students may arrive at school late and be marked absent or simply wander around the school premises, but yet marked as being present. However, the incrementally high rates of school truancy, along with wide and far-reaching life consequences of this social and public health issue, suggest the need for further research to better understand truancy and truant youth from a South African perspective.

The purpose of this article is to review extant South African and international research on school truancy studies with the aim to reflect on conceptual issues, causes of truant behaviour and on current research trends as well as its implications for future truancy intervention and policy making. Furthermore, it is also envisaged that the implications of this review will provide evidence, guidance as well as some caution for future researchers who wish to advance the study of truant behaviour among students.

\section{Conceptual Issues}

Among the key issues when focussing on school absenteeism and truancy, is to understand precisely the meaning and definition of these concepts. Reid (2010) sheds light on this issue by referring to various types of truancy. These include deliberately missing school without good cause. A range of various forms of school absenteeism can also be classified as truancy. These include specific lesson absence or specific lesson truancy, post-registration absence or post-registration truancy, psychological absence or psychological truancy and most controversially parentally condoned absence and parentally condoned truancy. Reid (2010) cited that in certain circles, specific lesson absence, post-registration absence and parentally condoned absence are regarded as not being truancy. Others disagree and equate 'being absent without good reason' with truancy, irrespective of its cause (Maynard, Salas-Wright, Vaughn \& Peters, 2012). It seems this is one of the reasons why operational definitions of truancy usually varied from study to study.

Other researchers held different views on exactly what constitute school truancy. For Stolls (1990), truancy can be regarded as being absent from school for no legitimate reason. Atkinson, Haysey, Wilkin \& Kinder (2000) introduced the concept of time into their definitions as they referred to differences in the extent of absences, from avoidance of a single lesson to truanting for several days, weeks or in rare cases, even months. O'Keef (1994) emphasized the challenges in classifying post-registration truancy and specific-lesson truancy, as these forms of absence are normally omitted from official school returns. Similarly, Kinder, Wakefield and Wilkin (1996) acknowledged that post-registration truants are not necessarily absent from school, as they may be hiding somewhere on the school premises in order to skip particular classes. The American National Centre for School Engagement proposed a brief and concise definition: any form of unexcused absence from school (Seeley, 2013).

However, more recently, truancy is commonly defined as excessive unexcused absence (Kim \& Page, 2013). Clinically, truancy has been categorised as a kind of conduct disordered behaviour along the same lines as stealing, lying, destructiveness and cheating (American Psychiatric Association, 2013). As an early childhood risk, school truancy is considered as part of a developmental pathway to more serious, later criminal careers. Chronic truanting students often miss opportunities to follow their school curricula, show low academic achievement and are likely to lose interest in school. School truancy, therefore should be considered an important marker of a child's social adjustment and specifically risk for future challenging behaviour.

Needless to say, it is for these reasons that official statistics on school truancy or unauthorised absence need to be treated with a great measure of circumspection. Official statistics often keep changing their own definitions and sometimes even the timing of data. However, whatever methods are employed to quantify pupils' absences from school, there is a growing concern that despite all the best efforts of schools and local and national education departments, global school attendance had not improved over the last thirty years. Recent evidence suggest that as many girls as boys currently engage in truant behaviour. More disturbing is that the onset of truancy is reportedly becoming younger, with approximately 36 percent of all truants beginning their patterns of irregular school and lesson attendance whilst at primary school (Reid, 2010). 


\section{Why do Truants Opt not to Attend School?}

There is increasing evidence that the phenomenon of truancy (i.e. unexcused absence form from school) is associated with a host of interconnected and overlapping negative individual, family, social and community risks factors (Ovink, 2011). Its causes continuously seem to be changing and are becoming increasingly complex. There are indications that research findings can vary depending upon the methodology used. For example, data obtained through methodology utilising school-based surveys, community surveys, pupils' self-referral instruments, parentally obtained information or teacher assessed questionnaires, often reveal significant differences in the outcomes on matters pertaining to the extent of parentally condoned absenteeism.

For Hayes (2011), home background and social circumstances were primary reasons for truancy. Reid (2010) in a detailed study of 128 persistent absentees and two matching control groups $(n=384)$ reported that school based factors such as bullying, the curriculum and poor teaching were the preponderant factors in a clear majority of cases. However, all individual cases to a greater or lesser extent contained aspects of social, psychological and institutional features, bearing in mind that an important aspect about truancy is that each case is largely unique in nature. In the aforementioned study conducted by Reid (2010), some related trends however were found in a high percentage of cases of truants presenting with lower levels of self-esteem and poorer academic self-concepts compared to their regular attending peers.

Other recent studies have found the causes of truancy to be directly linked to the lack of child-rearing skills among parents or carers (Donoghue, 2011) and the effects of dysfunctional local communities (Hong, Algood, Chiu and Lee, 2011). Henry (2010) found that the prime causes were personal, family, school and community based factors. Individual aspects included issues like - lack of self-esteem, social skills and confidence; poor peer-group relationships; lack of academic ability; special needs; lack of concentration and self-management skills. Family factors included parentally condoned absences, not valuing education, domestic challenges, inconsistent or inadequate parenting and economic deprivation. Community issues revolved around socio-economic factors, locations, housing, local attitudes, culture, criminality, vandalism and a sense of feeling safe. Within schools, the main issues were poor management, the ease at which some pupils could slip away unnoticed, poor teacher - pupil relations, the school 'ethos', a perceived irrelevance of some aspects of the national curriculum, bulling and poor learning-teaching strategies.

\section{Prior Reviews of Truancy Studies and Interventions}

A number of prior reviews have synthesized knowledge on truancy and interventions to combat this kind of learner behaviour pattern and improve school attendance. Most of these reviews have been narrative in nature and have not presented their finding systematically, often reviewing the same programs, emphasizing particular studies considered 'effective` (Maynard, McCrea, Piggot and Kelly, 2013). Klima, Miller and Nunlist (2009) undertook a meta-analysis of 22 experimental and quasi-experimental studies which aimed at evaluating the effects of dropout and truancy interventions. These authors reported small positive impacts on dropping out, achievement and attendance. For attendance and enrolment outcomes, it was reported that alternative education programs, behavioural programs and school based mentoring programs were the modalities found to be most effective.

Sutphen, Ford \& Flaherty (2010) conducted a systematic review of the effects of truancy interventions. Their review included 16 studies of truancy intervention studies published in peer-reviewed journal between 1990 and 2007 and comprised experimental, quasi-experimental and single group pretest-post-test studies and a broad range of intervention modalities including universal, selective and indicated programs. The authors of this review highlighted a paucity of truancy intervention research and a lack of consistency in definitions of truancy used by researchers. They also identified individual interventions that demonstrated beneficial effects, including interventions using contingency management, group guidance and parental notification as well as some community based and collaborative interventions.

In their study, Maynard, Sala-Wright, Vaughn and Peters (2012), explored the presence of heterogeneity among truant youth to provide a more nuanced examination of the nature of adolescent truancy and examine distinct profiles of truant youth as they relate to externalising behaviours. Latent profile analysis was employed to examine the heterogeneity of truant youth by using a nationally representative sample of 1,646 truants. Five key indicator variables were utilized to identify latent classes: school engagement, participation in school-based activities, grades, parental academic involvement and number of school days skipped. Additionally, multinomial regression was employed to examine the relationship between latent truant youth classes and externalizing behaviours. Four classes of truant youth were identified: achievers (28.55\%), moderate students (24.30\%) academically disengaged (40.89\%) and chronic truants (6.26\%). Based on the findings of this study, it emerged that group membership was associated differentially with 
marijuana use, fighting, theft and selling drugs. It was also found that truants are not a homogenous group, but rather presents with different risk profiles as they relate to key indicators, demographic characteristics and externalizing behaviours.

In a study of limited scope conducted by Sheppard (2009), data was collected of 57 students' which measured their attitudes to school and schoolwork and their perceptions of their parents' involvement in their education. The author examined the role of these variables in relation to school attendance, 'good' and 'poor' attenders of 12 and 13 years of age and compared it on a number of quantifiable measures regarding their perceptions of schoolwork and their parents' behaviour in relation to aspects of their schooling.

The findings of this study suggested that good, medium and poor school attenders avoided class if possible, but good attenders were more likely to do their homework and perceived their parents as more involved in their education. Further differences between the groups showed that most of the students, irrespective of the group which they were in, indicated that they did their school work when it was easy and fun. However, more students in the good attending group gave a reason that suggested that they understood the importance of education particularly for career opportunities. The fact that such an attitude was expressed in response to an open-ended question suggested that it was salient for them and may have represented a reflection of their parents' values. The results regarding homework suggested that generally parents were perceived as acknowledging the importance of homework, although medium and low attenders were more likely to defy parental instruction to do homework. Therefore, while all groups avoided class if they could, good attenders more often obeyed parental requests to do homework assignments. If this is a reliable result, it may reflect children's perception of parental involvement in their education and their learning. In addition, poor attenders and truants, not having completed their homework, would perhaps have a reason to be absent from school or skip particular lessons, most likely being the absence of their parents' interest and involvement in their schooling.

Monobe and Baloyi (2012) cited that with the emergence of the democratic South Africa, a number of disciplinary problems emerged, of which student truant behaviour is one of the most prominent. Yet, there appears to be a gap in the literature which report on the phenomenon of truancy and intervention programmes to improve school and lesson attendance by students in South Africa compared to the international context. In the section which follows, truancy studies conducted in South Africa will be focussed on.

Based on a study conducted by Masithela (1992), it was found that learners tend to miss lessons during the first and second periods as well as during the last five periods of the school day. The tendency of missing certain classes towards the end of the school day shows that some of "hidden truancy" is prevalent, and that some students are marked present in the attendance register, but fails to attend all lessons. On the other hand, they come late and are marked absent or be somewhere on the school premises not attending certain lessons, but still be marked as being present in the attendance registers (Smith, 1996).

In her study of the nature of truancy and to explore the life world of truants in secondary schools, Moseki's (2004) investigation was undertaken with a sample of 758 Grade 10 students from three secondary schools which were randomly selected out of 14 secondary schools in the town of Kimberley in the Northern Cape Province of South Africa. Data was collected by means of a questionnaire which was completed by the sample of participants during the first period on the day of the investigation. Teachers, who were responsible for either class registers or teaching a lesson at the time, assisted with the distribution of the questionnaires and supervised the learners as they completed the questionnaire.

Based to the main findings of this study, it seemed that truancy appears to be a universal phenomenon which is not restricted to students from one particular socio-economic background. Other significant findings demonstrated the following: more male learners than female learners engage in truancy; of the subjects or learning areas that learners tend to skip often Mathematics and Life Science, top of the list; learners whose parents are readily involved in school activities, would seldom or never engage in truancy, while those whose parents are seldom or never involved have always skipped certain classes. Finally, significantly more learners who have a good relationship with teachers indicated that they never skipped school or any specific lessons, while the reverse clearly seemed to be the case for learners who had poor relationships with authoritative figures in their learning environment (Moseki 2004).

A more recent South African truancy study conducted by van Breda (2006) aimed at addressing the following question: How can teachers, in loco parentis, be equipped with the necessary skills and resources to deal with the issue of truant behaviour among early adolescent learners. The empirical investigation was carried out through quantitative as well as qualitative research methodology. A focus group interview was conducted with 6 learners affording them an opportunity to express their perceptions and experiences as truants. Interviews were conducted with managers of schools in the area where the study was conducted to obtain their views with regards to truant behaviour among students who attended their respective schools. A questionnaire which investigated truancy related aspects among adolescent learners such as interaction with peers, parents and caregivers' involvement in learners' school work and learners' self-esteem 
regarding their schooling was administered to 300 randomly selected Grade 8 and 9 male and female students in the Metro East Education District of the Western Cape Province of South Africa.

It emerged from the data analysis of this study that $66.33 \%$ of the respondents who participated have demonstrated that they have engaged in truant behaviour since attending secondary school. The balance of the respondents indicated that they have not yet truanted in any manner. The following are some of the recurring themes which emerged from respondents' qualitative responses to the open-ended question included in the questionnaire:" Have you ever truanted ("bunked") any of your classes?" The following themes emerged from the responses of the participants who responded affirmatively to an open-ended question in the questionnaire as their reasons for engaging in truant behaviour:

Teacher ill-treatment of students including unfairness, undue corporal punishment administered by teachers; marginalisation of certain learners by their teachers; an uncaring and unsympathetic attitude displayed towards students by teachers and authoritative figures at school; perceived discriminatory behaviour demonstrated by some educators against learners; being insulted and 'picked upon' by certain teachers; truants viewing themselves as outcasts who were rejected at school by certain teachers and fellow learners, causing them to feel unhappy and unwelcome in their learning environment; dysfunctional family lives, lack of parental interest in their scholastic activities and no moral support received at home and embarrassment about physical appearance particularly among girls who felt that they didn't always look presentable enough to go to school.

\section{Interventions to Increase Student Attendance or Reduce Truancy}

In the light of the fact that truancy is a recognised problem among various disciplines - including education, psychology, social work, sociology, criminal justice and others - not only the conceptualisation of the problem, but also the approaches used to intervene are diverse. Emanating from a review of a number of interventions designed to increase student school attendance, it appears that interventions targeting school attendance seem to fall into several different categories and are delivered through a variety of modalities. Interventions generally target individual risk factors, such as school anxiety or phobia, low esteem, poor social skills, family factors, lack of parental involvement and school factors including school climate inconsistent attendant policies and poor relationships between teachers and students (Maynard, Sala-Wright, Vaughn and Peters (2012).

In addition to the variety of risk factors targeted, interventions also differ in terms of the settings in which they are implemented. Interventions have been implemented in clinical and community agency settings, schools, courts and police agencies. Interventions are also conducted as part of a collaborative effort between community agencies, schools, courts or by a single entity.

Due to the small number of studies in this synthesis, and the heterogeneous nature of the indicated studies, it is the view of the author that the findings from this review can provide some evidence and guidance, as well as some caution, for those who are concerned about, and trying to take action and develop policy to improve attendance of truant students. On the other hand, despite the increased pressure for evidence-based practice and policy and the serious and widespread problem of truancy, there continues to be a paucity of research in the area of interventions to improve school and class attendance for truants. Although more research is needed, more of the same is not suffice. Furthermore, many of the studies included in this review were plagued with methodological shortcomings and a number of gaps in the evidence base were identified. In the light of the afore-mentioned, recommendations to improve the quality and fill gaps in truancy research are discussed below.

\section{Recommendations to Improve Truancy Study Quality}

Due to the inherent limitations to single, group and pre-post test design studies, it is recommended that future research evaluating outcomes of interventions utilize a comparison group design, preferably with random assignment to limit other potential confounds. Should a single group pre - post-test design be utilized, researchers should not overstate their findings, discuss the limitations of the design and replicate their intervention and evaluate the outcomes utilizing a comparison group design.

To address the overall lack of adequate description of the intervention strategies, it is recommended that future research include detailed descriptions of the intervention to allow for replication. Descriptions should include details of each of the components of the intervention, the duration of each of the components, who implemented each of the components, the cost and funding of the intervention. In addition it is also recommended that researchers and authors clearly state their involvement in the development or implementation of the intervention. 
Keeping attrition to a minimal is important. For future research it is strongly recommended that researchers take attrition into account when designing the study and develop plans to mitigate potential threats to participant dropout. If there are participants who did not complete the program or dropped out from the research, a comparison between completers and non-completers should be provided and any statistically significant differences should be explained and taken into account.

Larger sample sizes are needed in future studies. It is recommended that when planning truancy studies and determining sample size researchers take into account potential challenges in gaining access and consent of parents and students, as well as anticipate mobility and dropout as the school year progresses. Researchers also need to take steps to ensure access to more complete student records and data.

Finally, it is strongly recommended that attendance be measured and reported in a consistent and clear way to allow for easier comparison across studies, as well as to allow for better transparency. Future research should report either attendance or absences in terms of a percentage of days absent or present; clearly specify the number of school days for which attendance was possible and the time period over which it was measured and measure both excused and unexcused absences as well as partial days absent and report these separately so that meaningful comparisons can be made across studies. In addition, it is recommended that researchers and authors present their findings in terms of clinical significance in addition to statistical significance.

\section{Conclusion}

There are numerous truancy interventions in operation with the goal of increasing attendance and it seems that many of these have been described in the literature as positively impacting the students and communities they are serving. Unfortunately, rigorous research to support truancy intervention is either not being conducted or is not being disseminated in a way that can inform others. Either way, evidence is not being built in a way that can add to the evidence base of effects of truancy interventions to inform practice and policy.

In order to move the field forward, the various disciplines engaged in truancy research need to take a critical look at barriers affecting research and dissemination. The social, political and practical issues and barriers will need to be acknowledged, examined and addressed if we hope to positively impact the attendance problem plaguing this country and others around the world.

\section{References}

American Psychiatric Association (2013). Diagnostic and statistical manual of mental disorders (5th ed.) Washington DC.

Atkinson, M., Haysey, K., Wilkin, A. \& Kinder, K. (2000). Raising attendance, Slough: NFER

Department of Basic Education (2010). Policy on Learner Attendance, Pretoria.

Donoghue, (2011). Truancy and the prosecution of parents: An unfair Burden on Mothers? The modern law review. Blackwell Publishing. Goldstein, J.S., Little, S.G., \& Akin-Little, A. (2003). Absenteeism: A review of the literature and school psychology's role. The California School Psychologist, 8, 127-139.

Hayes, D.H.(2011). Predicting Parental Home and Social Involvement in High School African American Adolescents. The High School Journal. The University of North Carolina Press.

Hendricks, MA., Sale, EW., Evans, C.J., McKinley L., \& Carter S.D. (2010). Evaluation of court intervention in four middle schools. Psychology in the Schools, 47(2).

Henry. K. L. (2010). Skipping school and using drugs: A brief report. Drugs: Education, Prevention, and Policy, 17, 650-657.

Hong, J.S., Algood, C.L., Chiu, Y.L., \& Lee, S.A. (2011). An Ecological understanding of Kinship Foster Care in the United States. Journal of Child and Family Studies. Springer Science and Business Media B.V.

Kim, H.Y., \& Page, T. (2013). Emotional Bonds with parents, Emotion Regulation and school related behaviour problems among elementary school truants. Journal of Child and Family Studies, 22, 869-878.

Kinder, K. Wakefield, A. \& Wilkin, A. (1996). Talking back: Pupil views on Disaffection, Slough: NFER.

Klima, T., Miller, M., \& Nunlist, C. (2009). What works? Targeted truancy and dropout programs in middle and high school. [Online] Available: http://www.wsipp.wa.gov/pub.asp (January 23, 2014).

Masithela, N.J. (1992. An investigation of the rolethat high-school teachers can play in reducing truancy in the Galeshewe area, with special reference to Tlhomelang High School. Unpublished MEd dissertation, University of KwaZulu Natal, South Africa.

Maynard, B.R., McCrae, K.T., Pigott, T.D., \& Kelly M.S. (2013). Indicated Truancy Interventions for Chronic Truant Students: A Campbell Systematic Review. Research on Social Work Practice 23 (1) 5-21.

Maynard, B.R., Salas-Wright., C.P., Vaughan M.G., \& Peters, K.E. (2012). Who are truant youth? Examining distinctive profiles of truant youth using latent profile analysis. Journal of Youth and Adolescence. Advance online publication.

Monobe, J.M., \& Baloyi, K. (2012). An investigation into some of the major causes f truancy in the Venda Technical College in the Limpopo Province. US-China Education Review. 1, 84-89. 
Moseki, M. (2004). The nature of truancy and the life world of truants in secondary schools. Unplublished MEd dissertation University of South Africa, Pretoria.

O'Keef, D.J. (1994). Truancy in English secondary schools. London; Sage.

Ovink, S.M. (2011). "This Aint My School!" Criminality, Control, and Contradictions in Institutional Responses to School Truancy. Quality Sociology, 34, 79-99.

Petrides, K.V., Chamorro-Premuzic,T., Frederickson, N., \& Furnham, A. (2005). Explaining individual differences in scholastic behaviour an achievement. British Journal of Educational Psychology, 75, 239-255.

Reid, K. (2010). Finding solutions to reduce truancy. Research in Education. Manchester University Press, Swansea Metropolitan University.

Reid, K. (2002). Truancy: Short and long term solutions. London, England: RoutledgeFalmer.

Reid, K. (1994). Dissatisfaction from school. London: Methnen and Co. Ltd.

Seeley, K. (2013). Guidelines for a national definition of truancy and calculating rates. [Online] http://www.schoolengagement.org (October 17, 2013)

Sheppard, A. (2009). School attendance and attainment: Poor attenders' perceptions of schoolwork and parental involvement in their education. British Journal of Special Education. 36 (2).

Smit, P. A. (1996). The life world of truants: Guidelines for the Educational Psychologist. Unpublished Med dissertation. Rand Afrikaans University, Johannesburg.

Stoll, P. (1990). 'Absent pupils who are officially present', Education today. 3, 22-30.

Sutphen, R. D., Ford, J .P., \& Flaherty, C. (2010). Truancy intervention: A review of the research literature. Research on Social Work Practice, 20, 161-171.

Valentine, J.C., Pigott, T.D., \& Rothstein, H.R. (2010). How many studies do you need? A primer on statistical power for meta-analysis. Journal of Education and Behavioural Statistics, 35, 215-247.

Van Breda, M.J. (2006). Guidelines for empowering secondary school educators, in loco parentis, in addressing truancy among early adolescent learners. Unpublished DEd thesis University of South Africa. Pretoria.

Valentine, J. C., Pigott, T. D., \& Rothstein, H. R. (2009). How many studies do you need? Aprimer on statistical power for meta-analysis. Journal of Education and Behavioural Staistics, 35, 215-247. 